\title{
Comparative study of the mutant prevention concentrations of vancomycin alone and in combination with levofloxacin, rifampicin and fosfomycin against methicillin-resistant Staphylococcus epidermidis
}

\author{
Li-guang Liu ${ }^{1,4}$, Yu-lin $\mathrm{Zhu}^{1,4}$, Li-fen $\mathrm{Hu}^{1}$, Jun Cheng ${ }^{1}$, Ying $\mathrm{Ye}^{1,2,3}$ and Jia-bin $\mathrm{Li}^{1,2,3}$
}

No mutant-prevention concentration (MPC) with methicillin-resistant Staphylococcus epidermidis (MRSE) has been reported. The study aimed to evaluate the propensity of vancomycin individually and in combination to prevent MRSE from mutation. A total of 10 MRSE clinical isolates were included in the study. Susceptibility testing demonstrated that the susceptibility rates to vancomycin, rifampicin, levofloxacin and fosfomycin were $100,100,50$ and $90 \%$, respectively. The fractional inhibition concentration indices (FICI) for vancomycin combined with rifampicin, levofloxacin or fosfomycin were $\geqslant 1.5$ but $\leqslant 2, \geqslant 1.5$ but $\leqslant 2$, and $>0.5$ but $\leqslant 1.5$, respectively, implying indifferent interactivity. The MPC with susceptible strains was determined to be the lowest antibiotic concentration inhibiting visible growth among $10^{10} \mathrm{CFU}$ on four agar plates $(9 \mathrm{~cm}$ in diameter) after a 72-h incubation at $37^{\circ} \mathrm{C}$. The MPCs were $16 \sim 32,>64, \geqslant 64$ and $4 \sim 16 \mu \mathrm{g} \mathrm{ml}^{-1}$ for vancomycin, rifampicin, fosfomycin and levofloxacin, respectively. The vancomycin MPCs of combinations with fosfomycin $\left(32 \mu \mathrm{g} \mathrm{ml}^{-1}\right)$, levofloxacin $\left(2 \mu \mathrm{g} \mathrm{ml}^{-1}\right)$ and rifampicin ( 2 or $4 \mu \mathrm{g} \mathrm{ml}^{-1}$ ) were $1 \sim 4,16 \sim 32$ and $16 \sim 32 \mu \mathrm{g} \mathrm{ml}^{-1}$, respectively. Against mutants selected by vancomycin, rifampicin, levofloxacin and fosfomycin individually, antibiotics had standard MICs of $2 \sim 4 \mu \mathrm{g} \mathrm{ml}-1$ for vancomycin, $>64 \mu \mathrm{g} \mathrm{ml}^{-1}$ for rifampicin, $4 \sim 8 \mu \mathrm{g} \mathrm{ml}^{-1}$ for levofloxacin and $\geqslant 64 \mu \mathrm{g} \mathrm{ml}^{-1}$ for fosfomycin. Thus single-step mutation can lead to resistance of MRSE to rifampicin, levofloxacin and fosfomycin, rather than non-susceptibility to vancomycin. Vancomycin-fosfomycin combination might be a superior alternative to vancomycin in blocking the growth of MRSE mutants, especially for high-organism-burden infections.

The Journal of Antibiotics (2013) 66, 709-712; doi:10.1038/ja.2013.87; published onine 28 August 2013

Keywords: combination therapy; drug resistance; mutant prevention concentration; MRSE

\section{INTRODUCTION}

Staphylococcus epidermidis has become a prevalent cause of nosocomial infections due to increasing use of broad-spectrum antimicrobials, immunosuppressants and biomaterials in the clinic, and methicillinresistant S. epidermidis (MRSE) has accounted for a majority of S. epidermidis nosocomial infections. ${ }^{1,2}$ To date, vancomycin still remains the drug of choice against MRSE infections. However, decreased vancomycin susceptibility in staphylococci (including heterogeneous and homogeneous vancomycin-intermediate staphylococci) has been reported all over the world. Though there are alternatives to vancomycin such as linezolid, daptomycin, tigecycline and quinupristin-dalfopristin, they are expensive and not easy to access, especially in developing countries. With a limited pool of available antimicrobial agents capable of treating MRSE infections, the suppression of further emergence of resistance is of vital importance.

The 'mutant selection window' hypothesis ${ }^{3,4}$ proposes a way to fight against the drug resistance from a new viewpoint. It postulates that maintaining antimicrobial concentration at infection sites within mutant selection window, a range between MIC and mutant prevention concentration (MPC) is expected to select for and enrich resistant mutants. MPC is defined as the minimal concentration required to inhibit the growth of the least susceptible single-step mutant and approximated experimentally as the lowest concentration that allows no colony growth when more than $10^{10}$ cells are applied to drug-containing culture medium. Maintaining antimicrobial concentration at infection sites above MPC or concurrent use of

${ }^{1}$ Department of Infectious Diseases, the First Affiliated Hospital of Anhui Medical University, Hefei, Anhui, China; ${ }^{2}$ Anhui Center for Surveillance of Bacterial Resistance, Hefei, Anhui, China and ${ }^{3}$ Institute of Bacterium Resistance, Anhui Medical University, Hefei, Anhui, China

${ }^{4}$ These authors contributed equally to this work.

Correspondence: Dr Y Ye, Department of Infectious Diseases, the First Affiliated Hospital of Anhui Medical University, Jixi road 218, Hefei, Anhui 230022, China. E-mail: yeying2@139.com

Received 9 June 2012; revised 13 December 2012; accepted 8 May 2013; published online 28 August 2013 
two antimicrobials at concentrations above MIC will block the amplification of mutants and final resistance because bacteria will have to acquire two concurrent mutations for growth, which rarely exists in vivo. Because MPC determinations usually apply an inoculum size of approximately $10^{10} \mathrm{CFU}$ containing potentially resistant mutant subpopulations, MPC is a susceptibility parameter that can measure better the relative potential for selection of resistant mutants by antimicrobials than traditional susceptibility testing.

Ideally, MPC should be determined for organism-antibiotic pairings for which spontaneous mutation is a common mechanism of resistance. Available data indicate that the resistance of staphylococci to vancomycin, ${ }^{5-7}$ levofloxacin, ${ }^{8}$ rifampicin ${ }^{9}$ and fosfomycin ${ }^{10}$ comply with the above mentioned principle. So far, no study of the MPC for MRSE has been published yet. This study aimed mainly to compare the mutant-prevention efficacies of vancomycin used alone and in combination with levofloxacin, rifampicin or fosfomycin. Rifampicin, levofloxacin and fosfomycin were chosen in this study because they are cheap and penetrate well where vancomycin penetrates poorly (for example, the lung, cerebrospinal fluid and bone joint). In addition, the susceptibility rates of $S$. epidermidis to fosfomycin ${ }^{11}$ and rifampicin ${ }^{12,13}$ remain fairly high.

\section{MATERIALS AND METHODS}

\section{Strains and drugs}

Totally 10 MRSE strains isolated from different clinical specimens (7 blood, 1 wound swabs, 2 catheter tips) were chosen for the study. These strains were from different patients ( 5 neonates, 2 elders, 2 adults, 1 child) hospitalized in three general hospitals in Hefei city, Anhui province, China. Specific identification of MRSE was conducted previously using intragenic primer sets for staphylococcus $16 \mathrm{SrRNA},{ }^{14}$ mecA gene sequences ${ }^{14}$ and $S$. epidermidis species-specific gene sequence. ${ }^{15}$ Staphylococcus aureus ATCC 29213 was stored in Anhui Center for Surveillance of Bacterial Resistance and used as the quality control strain in MIC determination and synergy testing. The antimicrobials in this study and their suppliers were as follows: levofloxacin (LVX), rifampicin (RIF) and fosfomycin (FOF) standards were obtained from the National Institute for the Control of Pharmaceutical and Biological Products (NICPBP; Beijing, China). Vancomycin (VAN) was purchased from Eli Lilly Japan K.K, Seishin Laboratories (Kobe, Japan). Glucose-6-phosphate was purchased from Sigma (Sigma, Deisenhofen, Germany).

\section{MIC determination}

The MICs were determined using the standard agar doubling dilution method. When MIC and MPC of fosfomycin or fosfomycin-containing combination were to be determined, Mueller-Hinton agar (MHA) should be supplemented with $25 \mu \mathrm{g} \mathrm{ml}^{-1}$ glucose-6-phosphate. ${ }^{16}$ ATCC 29213 (S. aureus) was included as the quality-control strain. An MRSE isolate was considered susceptible, intermediate or resistant for respective MIC values of $\leqslant 4,8-16$ or $\geqslant 32 \mu \mathrm{g} \mathrm{ml}^{-1}$ for vancomycin, $\leqslant 1,2$ or $\geqslant 4 \mu \mathrm{g} \mathrm{ml}^{-1}$ for levofloxacin, and $\leqslant 1,2$ or $\geqslant 4 \mu \mathrm{g} \mathrm{ml}^{-1}$ for rifampicin, according to the CLSI guidelines. ${ }^{16}$ An MRSE isolate was considered susceptible or resistant for fosfomycin MIC values of $\leqslant 32$ or $>32 \mu \mathrm{g} \mathrm{ml}^{-1}$, respectively, according to EUCAST guidelines ${ }^{17}$ (the breakpoint concentrations of fosfomycin against staphylococci are not available in the CLSI guidelines).

\section{Synergy testing}

Checkerboard synergy testing was performed for all the susceptible strains with two-fold agar dilutions on MHA. ${ }^{18}$ MHA was also supplemented with $25 \mu \mathrm{g} \mathrm{ml}^{-1}$ glucose-6-phosphate for synergistic testing of the fosfomycinvancomycin combination. The concentrations were set according to the MIC values of the preliminary susceptibility tests. To be exact, vancomycin concentrations (doubling dilutions) ranging $1 / 64-4 \times$ MIC in the columns and rifampicin, levofloxacin or fosfomycin concentrations (doubling dilutions) ranging 1/64-4 $\times$ MIC along the rows were combined with each other on the agar plate in a checkerboard style. The MIC of individual drug was tested again in parallel along the row or column. Agar plates were inoculated using a multipoint inoculator $\left(5 \times 10^{4} \mathrm{CFU}\right.$ per spot) and incubated at $37^{\circ} \mathrm{C}$ for $24 \mathrm{~h}$ The standard quality-control strain ATCC 29213 was incubated with each run Fractional inhibitory concentration (FIC) was calculated as the MIC of a drug in combination divided by the MIC of the drug alone, and the FIC index (FICI) was obtained by adding the FICs. The results of FIC indices were interpreted as synergistic $(\mathrm{FICI} \leqslant 0.5)$, indifferent $(0.5<\mathrm{FICI}<4)$ and antagonistic $(\mathrm{FICI} \geqslant 4)$ according to the most relevant criteria. ${ }^{19}$

\section{MPC determination and MIC retesting of putative mutants}

MPCs were determined as described by Zhao et al. ${ }^{20}$ Briefly, the tested bacteria were cultured in Mueller-Hinton broth and cultured for $24 \mathrm{~h}$. The suspension was centrifuged ( $4000 \mathrm{~g}$ for $10 \mathrm{~min}$ ) and re-suspended in Mueller-Hinton broth to yield a concentration of about $3 \times 10^{10} \mathrm{CFU} \mathrm{ml}^{-1}$. Finally, each agar plate containing known antibiotic concentrations was inoculated with about $3.0 \times 10^{9} \mathrm{CFU}$ of tested bacteria ( $100 \mu \mathrm{l}$ bacterial suspension), with four plates $(9 \mathrm{~cm}$ in diameter) for a given antibiotic concentration. The MPC was determined to be the lowest antibacterial concentration that completely inhibited visible bacterial growth among $10^{10} \mathrm{CFU}$ after $72 \mathrm{~h}$ incubation at $37^{\circ} \mathrm{C}$ in ambient air. Different doubling dilution concentrations were prepared for each antibiotic-bacteria combination, with $1 \times$ MIC as the lowest concentration. The top concentrations prepared for MPC determinations were 64,64 and $16 \mu \mathrm{g} \mathrm{ml}^{-1}$ for vancomycin, rifampicin and levofloxacn, respectively, which were all above the attainable maximum serum concentrations at the currently recommended doses (the corresponding dosing regimens are $1000 \mathrm{mg}$ i.v. twice daily for vancomycin, 450 or $600 \mathrm{mg}$ po or i.v. once daily for rifampicin, and 400, 500 or $750 \mathrm{mg}$ po or i.v. once daily for levofloxacin) The top concentration prepared for fosfomycin MPC determination was $64 \mu \mathrm{g} \mathrm{ml}^{-1}$, which is double the resistant or susceptible breakpoint concentration of Staphylococcus to fosfomycin $\left(32 \mu \mathrm{g} \mathrm{ml}^{-1}\right)$ according to EUCAST. ${ }^{17}$ The concentrations of levofloxacin $\left(2 \mu \mathrm{g} \mathrm{ml}^{-1}\right)$ and rifampicin (2 or $4 \mu \mathrm{g} \mathrm{ml}^{-1}$ ) used in combination with vancomycin reflect their mean serum concentrations at steady state in healthy adults ${ }^{21}$ and adult pulmonary tuberculosis patients, ${ }^{22,23}$ respectively. The concentration of fosfomycin $\left(32 \mu \mathrm{g} \mathrm{ml}^{-1}\right)$ used in combination with vancomycin equals the susceptible breakpoint concentration of staphylococci but is far below the mean serum concentrations at steady state according to currently recommended dosing regimens. ${ }^{24}$ It should be mentioned that MPCs were determined only for susceptible isolates. Independent cells recovered from the highest antibiotic concentrations after $72 \mathrm{~h}$ incubation were passaged five times on drug-free MHA. Antibiotic MICs against putative mutants were retested before and after passage on drug-free media. If any antibiotic MIC against mutants selected from 1/2 MPC was below the resistant breakpoint concentration, MHA plates containing antibiotic concentrations of $50 \% \times, 60 \% \times, 70 \% \times, 80 \% \times$, $90 \% \times$ and $100 \% \times$ MPC were prepared and inoculated with $1.2 \times 10^{10}$ cells of MRSE to see whether higher antibiotic MIC against mutants (doubling dilutions) would result. Retesting putative mutant for susceptibility should be accompanied by MIC retesting of its parental strain for comparison.

\section{RESULTS}

All MICs were conducted in triplicate on separate days. Table 1 lists MICs of the four antimicrobial agents mentioned above against 10 strains of MRSE. The susceptibility rates of these strains to vancomycin, rifampicin, levofloxacin and fosfomycin were $100 \%, 100 \%$, $50 \%$ and $90 \%$, respectively.

The FICI of vancomycin combination with rifampicin, levofloxacin or fosfomycin were $\geqslant 1.5$ but $\leqslant 2$ for 10 parental strains, $\geqslant 1.5$ but $\leqslant 2$ for 5 parental strains and $>0.5$ but $\leqslant 1.5$ for 9 parental strains, respectively.

All MPC determinations were made in duplicate and the results were identical. Table 2 reflects the MPCs of antimicrobials alone and in combination with vancomycin against MRSE isolates. Retesting putative mutants selected by individual antibiotics for susceptibility demonstrated that antibiotics had standard MICs of $>64 \mu \mathrm{g} \mathrm{ml}^{-1}$ for rifampicin, $4-8 \mu \mathrm{g} \mathrm{ml}^{-1}$ for levofloxacin and $\geqslant 64 \mu \mathrm{g} \mathrm{ml}^{-1}$ for 
Table 1 MICs of four antimicrobials against 10 strains of MRSE $\left(\mu \mathrm{g} \mathrm{ml}^{-1}\right)$

\begin{tabular}{|c|c|c|c|c|}
\hline \multirow[b]{2}{*}{ Isolate No. } & \multicolumn{4}{|c|}{ MIC for } \\
\hline & VAN & $R I F$ & $L V X$ & FOF \\
\hline 1 & 1 & 0.008 & 0.25 & 16 \\
\hline 2 & 1 & 0.008 & 0.25 & 0.25 \\
\hline 3 & 0.5 & 0.008 & 0.25 & 0.5 \\
\hline 4 & 0.5 & 0.008 & 2 & 1 \\
\hline 5 & 0.5 & 0.008 & 8 & 0.5 \\
\hline 6 & 0.5 & 0.008 & $>32$ & 1 \\
\hline 7 & 0.5 & 0.015 & 8 & 64 \\
\hline 8 & 1 & 0.008 & 0.125 & 2 \\
\hline 9 & 0.5 & 0.008 & 0.5 & 1 \\
\hline 10 & 0.5 & 0.015 & $>16$ & 1 \\
\hline
\end{tabular}

Abbreviations: FOF, fosfomycin; LVX, levofloxacin; MRSE, methicillin-resistant Staphylococcus epidermidis; RIF, rifampicin; VAN, vancomycin.

Table 2 MPCs of antimicrobials alone and in vancomycin combination against 10 strains of MRSE $\left(\mu \mathrm{g} \mathrm{ml}^{-1}\right)$

\begin{tabular}{|c|c|c|c|c|c|c|c|}
\hline \multirow[b]{2}{*}{ Isolate No. } & \multicolumn{7}{|c|}{$M P C$ for } \\
\hline & VAN & FOF & $V A N+F O F$ & $L V X$ & $V A N+\angle V X$ & $R I F$ & $V A N+R I F$ \\
\hline 1 & 32 & $>64$ & 16 & 8 & 32 & $>64$ & 32 \\
\hline 2 & 32 & $>64$ & 1 & 4 & 16 & $>64$ & 32 \\
\hline 3 & 32 & 64 & 2 & 8 & 32 & $>64$ & 32 \\
\hline 4 & 16 & 64 & 2 & $N T^{a}$ & $\mathrm{NT}^{\mathrm{a}}$ & $>64$ & 16 \\
\hline 5 & 32 & $>64$ & 16 & $N T^{a}$ & $\mathrm{NT}^{\mathrm{a}}$ & $>64$ & 32 \\
\hline 6 & 16 & 64 & 4 & $N T^{a}$ & $\mathrm{NT}^{\mathrm{a}}$ & $>64$ & 16 \\
\hline 7 & 16 & $N T^{a}$ & $N T^{a}$ & $N T^{a}$ & $N T^{a}$ & $>64$ & 16 \\
\hline 8 & 32 & $>64$ & 2 & 4 & 32 & $>64$ & 32 \\
\hline 9 & 32 & 64 & 4 & 16 & 32 & $>64$ & 32 \\
\hline 10 & 32 & $>64$ & 4 & $N T^{a}$ & $N T^{a}$ & $>64$ & 16 \\
\hline
\end{tabular}

Abbreviations: FOF, fosfomycin; LVX, levofloxacin; MPC, mutant-prevention concentration; MRSE, methicillin-resistant Staphylococcus epidermidis; RIF, rifampicin; VAN, vancomycin. NT, not tested.

fosfomycin. In contrast, against mutants selected by vancomycin individually, vancomycin had MICs of $2 \mu \mathrm{g} \mathrm{ml}^{-1}$ (for parental isolates 2-4 and 6-10) or $4 \mu \mathrm{g} \mathrm{ml}^{-1}$ (for parental isolates 1 and 5). Higher vancomycin concentrations than $1 / 2 \mathrm{MPC}$ (that is, $60 \% \times, 70 \% \times$, $80 \% \times$ or $90 \% \times$ MPC) did not result in higher vancomycin MIC against selected mutants (doubling dilutions). All the antibiotic MIC values against selected mutants remain unchanged before and after 5 consecutive days of passaging on drug-free agar plates. Antibiotic MICs against the parental strains remained the same as previously determined.

\section{DISCUSSION}

The inoculum effect here refers to antibiotic efficacy being impaired by large inocula (density), such as density-dependent declines in the rate and extent of antibiotic-mediated killing and increases in MIC. ${ }^{25}$ There have been other in vitro and animal studies that support the inoculum effect. ${ }^{26-28}$ Likewise, MPC increases with bacterial density because of the inoculum effect. ${ }^{29}$ Thus, antibiotic MPCs determined on agar plates are likely to be higher than the standard low-inoculum antibiotic MIC against the least susceptible single-step mutant-that is, MPC should be regarded as the actual MIC rather than the theoretically defined standard low-inoculum MIC against the least susceptible single-step mutants.

The findings indicate that single-step mutation cannot result in vancomycin non-susceptibility in MRSE (MIC $>4 \mu \mathrm{g} \mathrm{ml}^{-1}$ ), which is consistent with previous studies and the fact that non-susceptibility of staphylococci to vancomycin arises rarely in the clinic. ${ }^{12,13,30}$ In contrast, single-step resistant mutants could be selected relatively easily by exposure to antibacterials other than vancomycin, which is consistent with the fact that resistance of staphylococci to rifampicin, levofloxacin or fosfomycin is relatively common in the clinic. However, it should be realized that two factors account for the fact that resistance selection in vivo is usually more difficult than that in vitro: one is that the antibiotic concentration in vivo is not high enough to select resistance, the other is that the immune system can kill mutants effectively. According to this study, infections with a large enough bacterial density (for example, deep-seated abscess and native valve endocarditis) might not be eradicated because of the inoculum effect unless vancomycin concentration at infection sites reaches the elevated MPCs, but such a target can hardly be accomplished at infection sites other than serum. Besides, mutants can be selected and enriched where vancomycin penetrates poorly (for example, the lung, cerebrospinal fluid and bone joint) and when vancomycin dosing is suboptimal (for example, for patients undergoing dialysis therapy). All these conditions make it necessary to apply combination therapy instead of monotherapy.

Fortunately, compared with vancomycin alone, combined application of vancomycin and fosfomycin $\left(32 \mu \mathrm{g} \mathrm{ml}^{-1}\right)$ resulted in seven MPC reductions by at least four-fold within the susceptibility range of S. epidermidis to vancomycin $\left(\leqslant 4 \mu \mathrm{g} \mathrm{ml}^{-1}\right)$, with the exceptions of isolates 1 and 5. Although vancomycin combination with fosfomycin showed indifferent interaction in synergy testing in this study and the previous study, ${ }^{31}$ considering mutant growth can be blocked by concurrent use of vancomycin and fosfomycin at concentrations below their respective MPCs, an additive protective effect of vancomycin plus fosfomycin combinations on the selection of resistant $S$. epidermidis may be proposed. This slows the emergence of vancomycin non-susceptibility because the selective enrichment of vancomycin-susceptible mutants (that is, precursors of vancomycin non-susceptible mutants) is inhibited. An obvious fact was that against the least susceptible single-step mutants selected by vancomycin from parental isolates 1 and 5, vancomycin had MICs identical to the susceptible breakpoint concentration of S. epidermidis to vancomycin. This might be the reason why the vancomycin MPCs against these two isolates were not reduced by more than two-fold in combination with fosfomycin. Surprisingly, vancomycin in combination with rifampicin ( 2 or $4 \mu \mathrm{g} \mathrm{ml}^{-1}$ ) or levofloxacin $\left(2 \mu \mathrm{g} \mathrm{ml}^{-1}\right)$ did not lead to significant lowering of the isolates' vancomycin MPCs in general. According to Zhanel et al. ${ }^{32}$ and Firsov et al., ${ }^{33}$ the MPC of an antibiotic could not be lowered when combined with a partner at a relatively lower concentration. Likewise, the failure of vancomycin MPCs to be significantly lowered when combined with levofloxacin or rifampicin might also result from the lower concentrations of levofloxacin and rifampicin used in combination (levofloxacin and rifampicin are both concentrationdependent antibiotics). The reason for such a phenomenon was unanimously explained as an emergence of cross resistance by Zhanel et $a .^{32}$ and Firsov et al. ${ }^{33}$ Here, we give a possible explanation from another perspective. Theoretically speaking, two-step mutations would be required for mutant growth once both drugs are kept above MIC. However, because of the inoculum effect, the actual MIC 
(not the standard low-inoculum MIC) for an antibiotic increases with bacterial density. Thus, single-step mutation would result in mutant growth if one drug is kept above its actual MIC, whereas the other is below its actual MIC.

A recent study using a biofilm model of methicillin-resistant S. aureus by Tang et al. ${ }^{34}$ has confirmed that the antibacterial activity of vancomycin combination with fosfomycin is superior to vancomycin individually and vancomycin in combination with rifampin. Similar conclusion might also be applicable for MRSE. Forrest and Tamura ${ }^{9}$ retrospectively analyzed previous studies in vitro and in vivo. They concluded that rifampicin combination therapy seems to have improved treatment outcomes when there is a loworganism-burden infection such as those with biofilms (that is, prosthetic joint infections and prosthetic valve endocarditis caused by methicillin-susceptible staphylococci) but in general does not offer any benefit over antibiotic monotherapy for high-organism-burden infections such as native valve endocarditis. Time-kill studies by Kang-Birken ${ }^{35}$ confirmed the bactericidal activity of vancomycin combination with levofloxacin mimicked that of vancomycin alone. All these studies do not contradict our study.

\section{CONCLUSION}

In all, monotherapy of rifampicin, levofloxacin or fosfomycin is not recommended for MRSE infections because they cannot block the proliferation of resistant mutants. Likewise, suboptimal dosing of vancomycin monotherapy can also result in enrichment of mutants and decreased susceptibility of MRSE to vancomycin. Concurrent use of fosfomycin and vancomycin is probably a superior alternative when and where vancomycin monotherapy is not sufficient, especially for high-organism-burden infections. Furthermore, available data indicate that the combinational application of vancomycin and fosfomycin alleviates the renal toxicity caused by vancomycin. ${ }^{24}$ In contrast, vancomycin combination with rifampicin or levofloxacin is not recommended for MRSE infections. Our conclusions need to be validated in vivo.

\section{CONFLICT OF INTEREST}

The authors declare no conflict of interest.

\section{ACKNOWLEDGEMENTS}

This work was supported by the National Natural Science Foundation of China (no. 81071394)

1 Bereket, W. et al. Update on bacterial nosocomial infections. Eur. Rev. Med. Pharmacol. Sci. 16, 1039-1044 (2012).

2 Piette, A. \& Verschraegen, G. Role of coagulase-negative staphylococci in human disease. Vet. Microbiol 134, 45-54 (2009).

$3 \mathrm{Karl}$, D. The mutant selection window and antimicrobial resistance. J. Antimicrob. Chemother. 52, 11-17 (2003)

4 Zhao, X. \& Drlica, K. Restricting the selection of antibiotic-resistant mutants: a genera strategy derived from fluoroquinolone studies. Clin. Infect. Dis. 33 (Suppl 3), S147-S156 (2001).

5 Srinivasan, A., Dick, J. D. \& Perl, T. M. Vancomycin resistance in staphylococci. Clin. Microbiol. Rev. 15, 430-438 (2002).

6 Szabó, J. hVISANISA: diagnostic and therapeutic problems. Expert Rev. Anti. Infect Ther. 7, 1-3 (2009)

7 Shoji, M. et al. walK and clpP mutations confer reduced vancomycin susceptibility in Staphylococcus aureus. Antimicrob. Agents Chemother. 55, 3870-3881 (2011).

8 Smith, H. J., Nichol, K. A., Hoban, D. J. \& Zhanel, G. G. Stretching the mutant prevention concentration (MPC) beyond its limits. J. Antimicrob. Chemother. 51, 1323-1325 (2003).
9 Forrest, G. N. \& Tamura, K. Rifampin combination therapy for nonmycobacteria infections. Clin. Microbiol. Rev. 23, 14-34 (2010).

10 Michalopoulos, A. S., Livaditis, I. G. \& Gougoutas, V. The revival of fosfomycin. Int J. Infect. Dis. 15, e732-e739 (2011)

11 Falagas, M. E. et al. Antimicrobial susceptibility of Gram-positive non-urinary isolates to fosfomycin. Int. J. Antimicrob. Agents 35, 497-499 (2010).

12 Zhao, C. et al. Antimicrobial resistance trends among 5608 clinical Gram-positive isolates in China: results from the Gram-Positive Cocci Resistance Surveillance program (2005-2010). Diagn. Microbiol. Infect. Dis. 73, 174-181 (2012).

13 Hope, R., Livermore, D. M., Brick, G., Lillie, M. \& Reynolds, R BSAC Working Parties on Resistance. Non-susceptibility trends among staphylococci from bacteraemias in the UK and Ireland, 2001-06. J. Antimicrob. Chemother. 62 (Suppl 2), ii65-ii74 (2008)

14 Zhang, K. et al. New quadriplex PCR assay for detection of methicillin and mupirocin resistance and simultaneous discrimination of Staphylococcus aureus from coagulasenegative staphylococci. J. Clin. Microbiol. 42, 4947-4955 (2004).

15 Martineau, F., Picard, F. J., Roy, P. H., Ouellette, M. \& Bergeron, M. G. Species-specific and ubiquitous-DNA-based assays for rapid identification of Staphylococcus epidermidis. J. Clin. Microbiol. 34, 2888-2893 (1996).

16 Clinical and Laboratory Standards Institute. Performance standards for antimicrobia susceptibility testing; twenty-second informational supplement. CLSI document M100-S22 32, 1-188 (2012)

17 European Committee on Antimicrobial Susceptibility Testing. Breakpoint tables for interpretation of MICs and zone diameters. Version 2.0 (2012). http://www.eucast.org/ clinical _breakpoints (5 January 2012, date last accessed).

18 Lozniewski, A., Lion, C., Mory, F. \& Weber, M. In vitro synergy between cefepime and vancomycin against methicillin-susceptible and -resistant Staphylococcus aureus and Staphylococcus epidermidis. J. Antimicrob. Chemother 47, 83-86 (2001).

19 Odds, F. C. Synergy, antagonism, and what the chequerboard puts between them. J. Antimicrob. Chemother. 52, 1 (2003)

20 Zhao, X., Eisner, W., Perl-Rosenthal, N., Kreiswirth, B. \& Drlica, K. Mutant prevention concentration of garenoxacin (BMS-284756) for ciprofloxacin-susceptible or -resistant Staphylococcus aureus. Antimirob. Agents Chemother. 47, 1023-1027 (2003).

21 Chien, S. C. et al. Pharmocokinetic profile of levofloxacin following once-daily 500 milligram oral or intravenous doses. Antimirob. Agents Chemother. 41, 2256-2260 (1997)

22 Ruslami, R. et al. Pharmacokinetics and tolerability of a higher rifampin dose versus the standard dose in pulmonary tuberculosis patients. Antimirob. Agents Chemother. 51, 2546-2551 (2007).

23 Ruslami, R. et al. Pharmacokinetics of antituberculosis drugs in pulmonary tuberculosis patients with type 2 diabetes. Antimirob. Agents Chemother. 54, 1068-1074 (2010).

24 Roussos, N., Karageorgopoulos, D. E., Samonis, G. \& Falagas, M. E. Clinical significance of the pharmacokinetic and pharmacodynamic characteristics of fosfomycin for the treatment of patients with systemic infections. Int. J. Antimicrob. Agents 34, 506-515 (2009).

25 Udekwu, K. I., Parrish, N., Ankomah, P., Baquero, F. \& Levin, B. R. Functional relationship between bacterial cell density and the efficacy of antibiotics. J. Antimicrob. Chemother. 63, 745-757 (2009).

26 Bhagunde, P. et al. Mathematical modeling to characterize the inoculum effect. Antimicrob. Agents Chemother. 54, 4739-4743 (2010).

27 Soriano, F., Santamaría, M., Ponte, C., Castilla, C. \& Fernández-Roblas, R. In vivo significance of the inoculum effect of antibiotics on Escherichia coli. Eur. J. Clin. Microbiol. Infect. Dis. 7, 410-412 (1988)

28 Soriano, F., Ponte, C., Santamaría, M. \& Jimenez-Arriero, M. Relevance of the inoculum effect of antibiotics in the outcome of experimental infections caused by Escherichia coli. J. Antimicrob. Chemother. 25, 621-627 (1990).

29 Quinn, B., Hussain, S., Malik, M., Drlica, K. \& Zhao, X. Daptomycin inoculum effects and mutant prevention concentration with Staphylococcus aureus. J. Antimicrob. Chemother. 60, 1380-1383 (2007).

30 Biavasco, F., Vignaroli, C. \& Varaldo, P. E. Glycopeptide resistance in coagulasenegative staphylococci. Eur. J. Clin. Microbiol. Infect. Dis. 19, 403-417 (2000).

31 Grif, K., Dierich, M. P., Pfaller, K., Miglioli, P. A. \& Allerberger, F. In vitro activity of fosfomycin in combination with various antistaphylococcal substances. J. Antimicrob. Chemother. 48, 209-217 (2001)

32 Zhanel, G. G. Mayer, M., Laing N. \& Adam, H. J. Mutant prevention concentrations of levofloxacin alone and in combination with azithromycin, ceftazidime, colistin (Polymyxin E), meropenem, piperacillin-tazobactam, and tobramycin against Pseudomonas aeruginosa. Antimicrob. Agents Chemother. 50, 2228-2230 (2006).

33 Firsov, A. A., Vostrov, S. N., Lubenko, I. Y., Portnoy, Y. A. \& Zinner, S. H. Prevention of the selection of resistant Staphylococcus aureus by moxifloxacin plus doxycycline in an in vitro dynamic model: an additive effect of the combination. Int. J. Antimicrob. Agents 23, 451-456 (2004).

34 Tang, $\mathrm{H}$. J. et al. In vitro efficacy of fosfomycin-containing regimens agains methicillin-resistant Staphylococcus aureus in biofilms. J. Antimicrob. Chemother 67, 944-950 (2012).

35 Kang-Birken, S. L. Comparative in vitro activity of vancomycin and levofloxacin in combination with rifampin against planktonic versus sessile cells of Staphylococcus epidermidis. Pharmacotherapy 20, 673-678 (2000). 